\title{
Counting Statistics for Mesoscopic Conductors with Internal Degrees Of Freedom
}

\author{
Christopher Birchall and Henning Schomerus \\ Department of Physics, Lancaster University, Lancaster, LA1 4YB, United Kingdom
}

(Received 29 April 2010; published 6 July 2010)

\begin{abstract}
We consider the transport of electrons passing through a mesoscopic device possessing internal dynamical quantum degrees of freedom. The mutual interaction between the system and the conduction electrons contributes to the current fluctuations, which we describe in terms of full counting statistics. We identify conditions where this discriminates coherent from incoherent internal dynamics and also identify and illustrate conditions under which the device acts to dynamically bunch transmitted or reflected electrons, thereby generating super-Poissonian noise.
\end{abstract}

DOI: 10.1103/PhysRevLett.105.026801

The probabilistic nature of quantum transport constitutes a fundamental source of current fluctuations, whose study provides detailed information about the carrier dynamics [1]. A comprehensive characterization of these fluctuations is provided by the framework of full counting statistics (FCS), which delivers a unifying perspective on the conductance $G=I / V$ (where $I$ is the current and $V$ is the bias voltage), the shot noise $P=2 e I F$ (where $F$ is the Fano factor, with $F=1$ signifying the Poissonian statistics of uncorrelated carriers), and higher-order stationary current correlations [2]. In the regime of single-particle electronic transport close to equilibrium, the Pauli principle fundamentally constrains the current fluctuations, which results in sub-Poissonian noise with Fano factor $F<1$. Repulsive interactions between the charge carriers generally result in a further reduction of the noise [3]. SuperPoissonian statistics can be achieved in strongly correlated systems [4] or when superconducting correlations or entanglement are present at some of the electronic sources $[1,5]$. The noise can also be enhanced via external means, e.g., by the presence of classical multistate fluctuators [6].

Many quantum conductors of present interest, such as molecules with different conformations and nanoelectromechanical systems, as well as a wide range of qubit proposals, possess internal quantum degrees of freedom which can be associated with multiple internal states of a mesoscopic device [see Fig. 1(a)]. These degrees of freedom are not directly associated with the charge carriers but cannot be considered external because of the backaction of the passing carriers. The purpose of this work is to demonstrate that these internal dynamics can be effectively probed via their influence on the counting statistics. We find that, even though the internal degrees of freedom do not introduce strong correlations in the conventional sense [7], they can result in super-Poissonian statistics with $F \gg 1$. The transport then takes the form of dynamically created trains of transmitted or reflected electrons. We identify conditions of ideal dynamical bunching and show that these can be realized in a model system, a coherent which-path interferometer [8,9] consisting of an Aharonov-Bohm ring
PACS numbers: 73.23. $-\mathrm{b}, 05.60 . \mathrm{Gg}, 72.70 .+\mathrm{m}, 73.50 . \mathrm{Bk}$

which is electrostatically coupled to an excess electron in a double quantum dot [see Fig. 1(b) and results in Fig. 2]. We also identify a regime where the transmitted bunches consist of exactly two electrons, with $F=2$.

These phenomena are identified by extension of previous works on counting statistics in passive systems, in particular, counting statistics of conductors coupled to quantum detectors $[10,11]$, and the wave-packet approach [12], which provides a convenient dynamical perspective on FCS. The resulting expression for FCS takes the form of a generalized propagation of the density matrix for the internal degrees of freedom. A key concept emerging from these expressions is the extent of coherence maintained throughout the evolution of the internal system dynamics, which manifests itself in electronic interference contributions associated to different dynamical histories of the internal system state.

Model and framework.-We consider electronic transport through a mesoscopic system with internal degrees of freedom, as schematically depicted in Fig. 1(a). The system possesses $N$ internal states $|n\rangle, n=1,2,3, \ldots, N$, which affect the transport of electrons passing through the system. In terms of creation and annihilation operators $\psi^{\dagger}$ and $\psi$ of electrons in momentum (k) and position (r) representation, a reference Hamiltonian representative of (a)

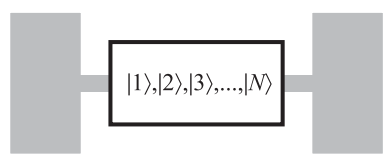

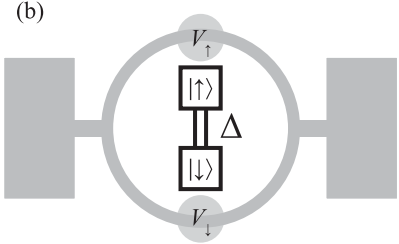

FIG. 1. (a) Sketch of a mesoscopic device with internal degrees of freedom, attached to leads contacted by electronic reservoirs. (b) Realization of such a system in terms of a coherent which-path interferometer, consisting of an excess electron in a double quantum dot (states $|\uparrow\rangle$ and $|\downarrow\rangle$ and tunnel splitting $\Delta$ ), which interacts via a state-dependent potential $V_{\uparrow, \downarrow}$ with charge carriers passing through an Aharonov-Bohm ring. 

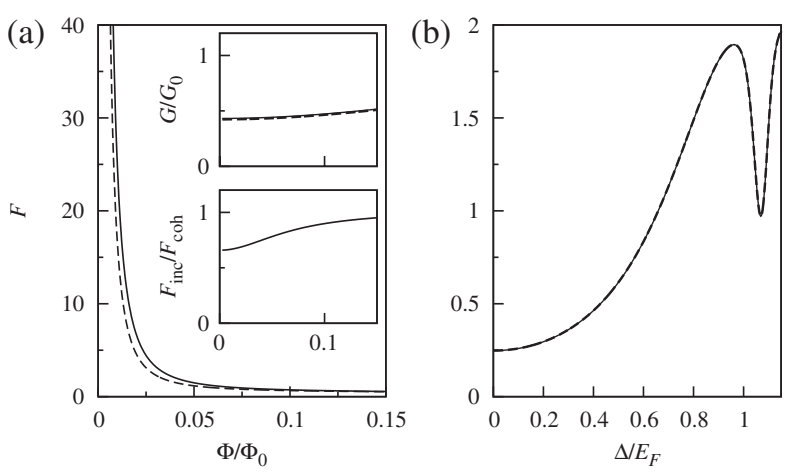

FIG. 2. Fano factor for the coherent which-path interferometer sketched in Fig. 1(b), where (a) shows results as a function of the magnetic flux $\Phi$ penetrating the Aharonov-Bohm ring, for fixed tunnel splitting $\Delta=0.01 E_{F}$, and (b) shows results as a function of $\Delta$, for fixed $\Phi=h / 2 e$. Solid (dashed) lines: Coherent (incoherent) internal dynamics. Insets in (a): Dimensionless conductance (top) and ratio of incoherent and coherent Fano factors (bottom). The results are obtained by propagation of minimaluncertainty wave packets with energy spread $\Delta E=0.007 E_{F}$. The interaction $V_{\uparrow, \downarrow}$ is modeled by an effectively impenetrable barrier of height $20 E_{F}$, which blocks one of the two arms (of length $L$, with $\hbar^{2} / 2 m L^{2}=0.061 E_{F}$ ) depending on the occupation of the double dot.

this problem takes the form

$$
\begin{aligned}
H= & \int d \mathbf{k} \epsilon(\mathbf{k}) \psi^{\dagger}(\mathbf{k}) \psi(\mathbf{k})+\sum_{n m} H_{n m}^{\mathrm{sys}}|n\rangle\langle m| \\
& +\sum_{n} \int d \mathbf{r} \psi^{\dagger}(\mathbf{r}) \psi(\mathbf{r}) V_{n}(\mathbf{r})|n\rangle\langle n| .
\end{aligned}
$$

Here, the first term describes the kinetic energy of electrons with dispersion relation $\epsilon(\mathbf{k})$, which is independent of the internal system state. The second term is the Hamiltonian of the isolated internal system degrees of freedom. The last term describes the interaction between these degrees of freedom and the electrons, which here is formulated in terms of a potential energy $V_{n}$ whose statedependent part is assumed to be confined to the scattering region (the potentials $V_{n}$ may also contain a stateindependent part outside this region, which can model, e.g., some features of the leads).

Note that we do not assume that the isolated system Hamiltonian commutes with the interaction part. Therefore, even when the internal degrees of freedom are initially prepared in an eigenstate $|\sigma\rangle$ of $H^{\text {sys }}$ (corresponding to energy $\mathcal{E}_{\sigma}$, where $\sigma=1,2,3, \ldots, N$ ), the interaction with scattered electrons will induce dynamics into the internal system. Moreover, since the system is not driven externally, total energy conservation implies that any change of internal system energy will be compensated by a change of energy of the transmitted or reflected electrons, which amounts to inelastic (but still coherent) scattering. We are interested in the consequence of these phenomena for the average current and its temporal fluctuations.
A general framework for the statistical description of the electronic transport is provided by FCS, which delivers information about the probability $P(Q ; \mathcal{T})$ that a number $Q$ of charge carriers are transmitted during a time interval $\mathcal{T}$. This information is encoded into the momentgenerating function

$$
\chi(\lambda)=\sum_{Q=0}^{\infty} P(Q ; \mathcal{T}) \exp (\lambda Q)=\sum_{k=0}^{\infty}\left\langle Q^{k}\right\rangle \frac{\lambda^{k}}{k !},
$$

where $\left\langle Q^{k}\right\rangle$ denotes the $k$ th moment of $Q$. Of particular interest are the associated cumulants of order $m$,

$$
\left\langle\left\langle Q^{m}\right\rangle\right\rangle=\left.\frac{\partial^{m}}{\partial \lambda^{m}} \ln \chi(\lambda)\right|_{\lambda=0},
$$

which for large times increase linearly in $\mathcal{T},\left\langle\left\langle Q^{m}\right\rangle\right\rangle \sim$ $(\mathcal{T} / \tau) q_{m}$. Here $1 / \tau$ is the attempt frequency of transmission for incoming electrons $(\tau=h / \mathrm{eV}$ for bias voltage $V$ ); the coefficient $q_{1}=g=G / G_{0}$ then delivers the dimensionless conductance (in units of the conductance quantum $G_{0}=e^{2} / h$ ), while $q_{2} / q_{1}=F$ delivers the Fano factor $[1,2]$.

General results.-In order to obtain a general expression for the moment-generating function, we adopt the wavepacket variant of the scattering approach, introduced in the absence of internal dynamics in Ref. [12]. We restrict our attention to circumstances where at most one electron at a time is interacting with the internal system. This corresponds either to weak interactions or to a small attempt frequency, where the latter can be realized either by applying a small bias voltage or by judiciously injecting sparse charge carriers via an electronic pumping device [13]. By exploiting the fact that under these conditions wave packets of consecutive electrons are nonoverlapping, the initial state of $\mathcal{N}$ electrons incident from the left lead can be formulated as the product of wave packets [14]

$$
\Psi_{\text {initial }}\left(\left\{\mathbf{r}_{n}\right\} ; t\right)=\left[\prod_{n=1}^{\mathcal{N}} \psi^{(\mathrm{in})}\left(\mathbf{r}_{n} ; t-t_{n}\right)\right] \sum_{\sigma} p_{\sigma}|\sigma\rangle,
$$

where $p_{\sigma}$ are the initial probability amplitudes of the internal system. Here, $t_{n} \approx n \tau$ denotes the arrival times of the electrons, which are spread out according to the attempt frequency, and $\mathcal{N} \approx \mathcal{T} / \tau$. The final state of the reflected or transmitted electrons then takes the form

$$
\Psi_{\text {final }}\left(\left\{\mathbf{r}_{n}\right\} ; t\right)=\sum_{\left\{\sigma_{n}\right\}_{n=0}^{\mathcal{N}}}\left[p_{\sigma_{0}}\left|\sigma_{\mathcal{N}}\right\rangle \prod_{n=1}^{\mathcal{N}} \psi_{\sigma_{n} \sigma_{n-1}}^{\text {(out) }}\left(\mathbf{r}_{n} ; t-t_{n}\right)\right]
$$

where

$$
\psi_{\sigma^{\prime} \sigma}^{(\mathrm{out})}(\mathbf{r} ; t)=r_{\sigma^{\prime} \sigma}(\mathbf{r} ; t)+t_{\sigma^{\prime} \sigma}(\mathbf{r} ; t)
$$

consists of a reflected and a transmitted wave packet, confined to the left and right lead, respectively; the indices indicate the final and initial internal states. These wave 
packets correspond to asymptotic transmission and reflection probabilities

$$
\begin{gathered}
R_{\sigma^{\prime} \sigma}=\int d \mathbf{r}\left|r_{\sigma^{\prime} \sigma}(\mathbf{r} ; t \gg 0)\right|^{2}, \\
T_{\sigma^{\prime} \sigma}=\int d \mathbf{r}\left|t_{\sigma^{\prime} \sigma}(\mathbf{r} ; t \gg 0)\right|^{2} .
\end{gathered}
$$

Following the general strategy of counting statistics developed for systems without internal degrees of freedom, we formulate the moment-generating function in terms of a generalized wave function: $\chi(\lambda)=\left\langle\Psi_{\text {final }}(\lambda) \mid \Psi_{\text {final }}(\lambda)\right\rangle$, where $\Psi_{\text {final }}(\lambda)$ is of the same form as Eq. (5) but with $\psi^{\text {(out) }}$ replaced by

$$
\psi_{\sigma^{\prime} \sigma}^{(\text {out })}(\mathbf{r} ; t, \lambda)=r_{\sigma^{\prime} \sigma}(\mathbf{r} ; t)+e^{\lambda / 2} t_{\sigma^{\prime} \sigma}(\mathbf{r} ; t) .
$$

Here, the counting field $\lambda$ serves for bookkeeping of the transmission events. After integration over the electron coordinates, the generating function takes the compact form of a matrix product:

$$
\chi(\lambda)=\mathbf{X}^{T} \mathcal{M}^{\mathcal{N}} \rho,
$$

where

$$
\begin{aligned}
\mathcal{M}_{\sigma^{\prime} \tilde{\sigma}^{\prime}, \sigma \tilde{\sigma}}= & \int d \mathbf{r} r_{\tilde{\sigma}^{\prime} \tilde{\sigma}}^{*}(\mathbf{r} ; t) r_{\sigma^{\prime} \sigma}(\mathbf{r} ; t) \\
& +e^{\lambda} \int d \mathbf{r} t_{\tilde{\sigma}^{\prime} \tilde{\sigma}}^{*}(\mathbf{r} ; t) t_{\sigma^{\prime} \sigma}(\mathbf{r} ; t)
\end{aligned}
$$

is the superoperator propagating the density matrix $\rho$ of the internal system state, generalized to include the counting field $\lambda$. Note that $\mathcal{M}$ can be interpreted as a matrix where each row and column is specified by two indices, with one index each arising from the propagation of the bra and the ket of the internal system state ( $\rho$ then corresponds to a vector). For the pure state specified in Eq. (4), the initial density matrix is $\rho_{\sigma \tilde{\sigma}}=p_{\sigma} p_{\tilde{\sigma}}^{*}$; with modification of this initial condition, the formalism also applies if the initial internal state is mixed. The vector $\mathbf{X}$ with components $\mathbf{X}_{\tilde{\sigma}^{\prime} \sigma^{\prime}}=\delta_{\tilde{\sigma}^{\prime} \sigma^{\prime}}$ embodies the trace of the final density matrix. In this dynamical description, the matrix elements $M_{\sigma^{\prime}, \sigma} \equiv \mathcal{M}_{\sigma^{\prime} \sigma^{\prime}, \sigma \sigma}$ are associated to the evolution of populations (the diagonal elements of $\rho$ ), while the other matrix elements describe corrections due to coherence (interference of electronic wave packets generated by different histories of the internal dynamics).

In practice, the internal degree of freedom will suffer from decoherence due to coupling to the environment. This can be described by a modified generalized propagator $\tilde{\mathcal{M}}=\mathcal{M} Z$, where the diagonal matrix $Z$ embodies suppression of off-diagonal elements of the intermediate density matrices. If the internal system state is continuously monitored, the internal dynamics become incoherent, $Z_{\tilde{\sigma}^{\prime} \sigma^{\prime}, \tilde{\sigma} \sigma}=\delta_{\tilde{\sigma}^{\prime} \sigma^{\prime}} \delta_{\tilde{\sigma} \sigma} \delta_{\sigma \sigma^{\prime}}$, and the time evolution reduces to a classical stochastic problem described by a rate equa- tion, which involves only transition probabilities between populations. The generating function then simplifies to

$$
\chi(\lambda)=\mathbf{Y}^{T} M^{\mathcal{N}} \mathbf{P},
$$

where

$$
M_{\sigma^{\prime} \sigma}=R_{\sigma^{\prime} \sigma}+e^{\lambda} T_{\sigma^{\prime} \sigma}
$$

is the classical propagator of the population probabilities with initial conditions $\mathbf{P}_{\sigma}=\left|p_{\sigma}\right|^{2}$, while the vector $\mathbf{Y}^{T}=$ $(1,1,1, \ldots)$ with unit components embodies the summation over all final states of the system [15].

Of particular interest is the steady state behavior of the system, corresponding to $\mathcal{T} \gg \tau$. To analyze this limit we diagonalize the matrices $\mathcal{M}$ (for coherent internal dynamics), $M$ (for incoherent internal dynamics), or $\tilde{\mathcal{M}}$ (for cases in between these two limiting scenarios) and denote the largest eigenvalue by $D_{\max }(\lambda)$. For large times, the cumulant-generating function then becomes

$$
\ln \chi(\lambda) \sim \frac{\mathcal{T}}{\tau} \ln D_{\max }(\lambda) .
$$

Therefore, the problem of full counting statistics is reduced to finding the eigenvalues of the matrices $\mathcal{M}, M$, or $\tilde{\mathcal{M}}$, respectively. By expanding $D_{\max }(\lambda)=\sum_{k=0}^{\infty} d_{k} \lambda^{k} / k$ ! as a Taylor series, the dimensionless conductance is given by $g=d_{1}$, and the Fano factor is given by $F=\left(d_{2}-d_{1}^{2}\right) / d_{1}$ [16].

Dynamical electron bunching.-As we now show, even though we consider electrons that never directly interact with each other, the internal system degrees of freedom can induce dynamical correlations which result in superPoissonian noise with $F \gg 1$, corresponding to dynamical bunching of electrons. This possibility is most transparently revealed in the case of incoherent dynamics of an internal two-state system. In anticipation of the concrete example discussed below, we denote the two internal states as $\sigma=+,-$. The matrix $M$ then takes the form

$$
M=\left(\begin{array}{ll}
R_{++}+e^{\lambda} T_{++} & R_{+-}+e^{\lambda} T_{+-} \\
R_{-+}+e^{\lambda} T_{-+} & R_{--}+e^{\lambda} T_{--}
\end{array}\right),
$$

and the largest eigenvalue can be calculated explicitly. Consider now the case of small switching probabilities $S_{+-}=R_{+-}+T_{+-} \ll 1, S_{-+}=R_{-+}+T_{-+} \ll 1$. On average, the two states are then populated with probabilities $P_{+}=S_{+-} /\left(S_{+-}+S_{-+}\right)$and $P_{-}=S_{-+} /\left(S_{+-}+\right.$ $\left.S_{-+}\right)$. In terms of these probabilities, the dimensionless conductance takes the simple form $g \approx P_{+} T_{++}+P_{-} T_{--}$ of a time-averaged transmission probability. By assuming $T_{++} \neq T_{--}$, the Fano factor

$$
F \approx \frac{1}{S_{+-}+S_{-+}} \frac{2 P_{+} P_{-}\left(T_{++}-T_{--}\right)^{2}}{P_{+} T_{++}+P_{-} T_{--}},
$$

on the other hand, can become arbitrarily large. The noise is then dominated by the rare probabilistic switching between states of different transmission probabilities. In the 
limiting case that one of the two transmission probabilities is much larger than the other (say, $T_{++} \gg T_{--}$), the device is partially transmitting during some time intervals but effectively shut down during others, which results in well spaced-out trains of transmitted electrons. These observations also extend to multistate dynamics; noise is generally enhanced whenever the transmission probabilities differ between the states of the device.

Application to a mesoscopic device.-We now illustrate how conditions of dynamically enhanced current fluctuations can be realized in a specific mesoscopic device, the coherent which-path interferometer shown in Fig. 1(b) $[8,9]$. The internal degree of freedom arises from an excess electron in a double quantum dot, with states $|\uparrow\rangle$ and $|\downarrow\rangle$ for occupation of the upper or lower dot, respectively. Depending on its location, this electron blocks the path of charge carriers moving through the upper or lower arm of an Aharonov-Bohm ring, where coherent transport can be tuned by varying the magnetic flux $\Phi$. As we are interested in situations where the isolated system Hamiltonian does not commute with the interaction term, we assume that the isolated system eigenstates are the symmetric and antisymmetric orbital states $| \pm\rangle=(|\uparrow\rangle \pm$ $|\downarrow\rangle) / \sqrt{2}$ of the excess electron, which are separated by a tunnel splitting energy $\Delta$ (corresponding to $\mathcal{E}_{ \pm}=\mp \Delta / 2$ ).

To characterize the transport through this device, we calculate the matrix elements (11) by numerical propagation of initially Gaussian wave packets. The conductance and Fano factor are then obtained from the eigenvalues of the matrices $\mathcal{M}(\lambda)$ (for coherent internal dynamics) and $M(\lambda)$ (for incoherent internal dynamics), as described above. Figure 2(a) shows the resulting Fano factor as a function of $\Phi$ for a set of system parameters. We observe that the current fluctuations can be significantly enhanced above the threshold $F=1$ for super-Poissonian noise. For $\Phi \rightarrow 0$, the switching probabilities $S_{+-}$and $S_{-+}$vanish because of the parity symmetry of the system [9], and the Fano factor increases over all bounds, as predicted by Eq. (16). In this regime, the Fano factor discriminates coherent from incoherent dynamics (bottom inset; as shown in the top inset, the conductance is unaffected by this distinction). In contrast, when half a flux quantum penetrates the ring $(\Phi=h / 2 e)$, parity symmetry ensures that each transmission event is strictly correlated with a transition of the internal system degree of freedom, while no such transitions occur when an electron is reflected (i.e., $R_{-+}=R_{+-}=T_{++}=T_{--}=0$ ) [9]. Even in this case, dynamical electron bunching can occur: The maximal Fano factor for incoherent internal dynamics now is $F=$ 2 , which is realized when $R_{--}, T_{-+} \ll R_{++}, T_{+-}$(or vice versa), so that $P_{+} \gg P_{-}$. The transport then consists of pairs of transmitted electrons, which coincide with the rare transitions into the state $|-\rangle$ (first transmitted electron), followed by its immediate depopulation (second transmitted electron). Figure 2(b) shows that such conditions can be met by tuning the tunnel splitting $\Delta$ and are insensitive to the degree of coherence in the internal dynamics. Guided by these results, we expect that super-Poissonian noise statistics should be common when mesoscopic systems possess internal degrees of freedom.

Concluding remarks.-In summary, we have investigated the phase coherent transport through mesoscopic devices with internal degrees of freedom and formulated the full counting statistics in terms of a generalized propagator of the density matrix for these freedoms [Eqs. (10)(14)]. In general, the counting statistics depend on the degree of coherence of the internal system dynamics. The dynamical switching of the internal system degrees of freedom can enhance the current fluctuations, resulting in Fano factors that significantly exceed the threshold $F=1$ for super-Poissonian noise.

[1] Ya. M. Blanter and M. Büttiker, Phys. Rep. 336, 1 (2000).

[2] Yu. V. Nazarov and Ya. M. Blanter, Quantum Transport: Introduction to Nanoscience (Cambridge University Press, Cambridge, England, 2009).

[3] D. A. Bagrets and Yu. V. Nazarov, Phys. Rev. B 67, 085316 (2003).

[4] D. A. Bagrets, Y. Utsumi, D. S. Golubev, and Gerd Schön, Fortschr. Phys. 54, 917 (2006); E. V. Sukhorukov, G. Burkard, and D. Loss, Phys. Rev. B 63, 125315 (2001).

[5] G. Burkard, D. Loss, and E. V. Sukhorukov, Phys. Rev. B 61, R16 303 (2000); V. Giovannetti, D. Frustaglia, F. Taddei, and R. Fazio, Phys. Rev. B 74, 115315 (2006).

[6] F. Hassler, G. B. Lesovik, and G. Blatter, arXiv:1001.0578.

[7] As considered, e.g., in J. Koch, F. von Oppen, and A. V. Andreev, Phys. Rev. B 74, 205438 (2006).

[8] H. Schomerus, Y. Noat, J. Dalibard, and C.W.J. Beenakker, Europhys. Lett. 57, 651 (2002).

[9] H. Schomerus and J.P. Robinson, New J. Phys. 9, 67 (2007).

[10] D. V. Averin and E. V. Sukhorukov, Phys. Rev. Lett. 95, 126803 (2005).

[11] G. Schaller, G. Kießlich, and T. Brandes, Phys. Rev. B 81, 205305 (2010); arXiv:1004.2604.

[12] F. Hassler, M. V. Suslov, G. M. Graf, M. V. Lebedev, G. B. Lesovik, and G. Blatter, Phys. Rev. B 78, 165330 (2008).

[13] L. P. Kouwenhoven et al., Phys. Rev. Lett. 67, 1626 (1991).

[14] Because wave packets are nonoverlapping, the analysis does not require us to antisymmetrize the wave function.

[15] The reduction from $\mathcal{M}$ to $M$ also occurs when inelastically scattered wave packets do not energetically overlap.

[16] The leading coefficient in this expansion is $d_{0}=1$, as follows from the normalization of the wave function $\Psi$. This eigenvalue corresponds to the left eigenvector $\mathbf{X}$ of the matrices $\mathcal{M}$ and $\mathcal{M}$, or $\mathbf{Y}$ for the matrix $M$, at $\lambda=0$. 